\title{
Numerical Simulation of Heat Transfer Diffusion- Convection in Two-Dimensional Space
}

\author{
Desy Purnami Singgih Putri \\ Information Technology \\ Department, \\ Faculty of Engneering, Udayana \\ University \\ Jimbaran, 80361, Indonesia
}

\author{
I Made Sunia Raharja \\ Information Technology \\ Department, \\ Faculty of Engneering, Udayana \\ University \\ Jimbaran, 80361, Indonesia
}

\author{
Kadek Suar Wibawa \\ Information Technology \\ Department, \\ Faculty of Engneering, Udayana \\ University \\ Jimbaran, 80361, Indonesia
}

\begin{abstract}
The diffusion convection equation is a mathematical model of transport event that occurs in the fluid. The equation can be solved numerically by using finite element method and in this research use Least Square finite element method. This method is based on minimizing second cube residue and on its application, it will produce a symmetric system and definite positive linear equations. This research will present a numerical simulation on the diffusion convection equation in two dimensional space use linear interpolation.
\end{abstract}

\section{General Terms}

Computational Mathematics, Algorithms, Numerical Simulation.

\section{Keywords}

finite element method, least square, convection, diffusion.

\section{INTRODUCTION}

The discussion about partial differential equations still continues and grow well in theory and application. The problem on mathematical modeling in other fields of science such as physics, engineering and many chemical fields uses partial differential equations [5]. One example of a nature phenomenon of mathematical modeling is the event of conductive heat transfer which is modeled into the heat equation.

The heat equation which is the parabolic equation can be solved analytically [1]. However, in some cases finding an analytical solution of heat equation is quite complex. Therefore, numerical methods are used to assist the solution. One of the most commonly used numerical methods in solving differential equations in general and the heat equations is finite difference methods. There are many finite element methods that can be used, one of them is the Alternating Direction Implicit Method (ADI).

In addition to conduction, the heat transfer also occurs by diffusion- convection in the fluid. This transport equation is then modeled into diffusion convection equation. In contrast to the conduction heat equation, the diffusion convection equation has a higher complexity. Method which is more commonly used for the fluid transport equation is the finite element method. Among some types of finite element methods, Least Squares method is more commonly used especially in the field of fluid mechanics and electromagnetics [2]. The Least Square finite element method is based on minimizing second rank residuals. As an illustration, note the differential equation
$B u=0$, at the limit $\Omega$

With $\mathrm{A}$ is a linear differential operator, $\mathrm{B}$ is a limit operator, $\mathrm{u}$ is a dependent vector, $\mathrm{f}$ is strength vector and $\Omega$ is a domain. First suppose the approximation solution of $\mathrm{u}$

$u \cong \phi_{j}(x) u_{j}, j=1,2, \ldots, n$

With $u_{j}$ is a parameter, $\phi_{j}(x)$ is a trial function and vector $x$ is an independent variable. Then look for a minimizer from

$$
I(u)=\int_{\Omega}(A u-f)^{2} d \Omega
$$

Then Least Square solution is calculated from the following variation equation

$$
\int_{\Omega}\left(A \phi_{i}\right)^{T}(A u-f) d \Omega
$$

Although the formulation on the Least Square method is simple, it provides significant benefits [6].

2. MATHEMATICS MODEL OF THE DIFFUSION CONVECTION EQUATION IN TWO-DIMENSIONAL SPACE

Based on the energy equation of [3]

$$
\rho c_{p}\left(\frac{\partial T}{\partial t}+u \frac{\partial T}{\partial x}+v \frac{\partial T}{\partial y}\right)=k\left(\frac{\partial^{2} T}{\partial x^{2}}+\frac{\partial^{2} T}{\partial y^{2}}\right)
$$

With

$c_{p}=$ specific heat at constant pressure

$k=$ thermal conductivity

$u=$ the velocity component in the $\mathrm{x}$ direction

$v=$ the velocity component in the y direction

Next, name the stream $q_{x}=\frac{\partial T}{\partial x}$ and $q_{y}=\frac{\partial T}{\partial y}$. Equation (1)

can be written into the system

$\rho c_{p} \frac{\partial T}{\partial t}+\rho c_{p} u \frac{\partial T}{\partial x}+\rho c_{p} v \frac{\partial T}{\partial y}-k \frac{\partial q_{x}}{\partial x}-k \frac{\partial q_{y}}{\partial y}=0$

$q_{x}-\frac{\partial T}{\partial x}=0$

$q_{y}-\frac{\partial T}{\partial y}=0$

$$
A u=f \text { in } \Omega \text {, }
$$




\section{LEAST SQUARE FINITE ELEMENT METHOD}

First, equation (2) is discriminated to the time variable by using the $\theta$ method to obtain

$$
A \mu=F
$$

With

$$
\begin{array}{r}
\mu=U^{n+1}-U^{n}, A \mu=A_{0} \mu+\theta \Delta t\left[A^{*}(\mu)\right], F= \\
-\Delta t\left[A^{*}\left(U^{n}\right)\right], A^{*}(\mu)=A_{1} \frac{\partial \mu}{\partial x}+A_{2} \frac{\partial \mu}{\partial y}+A_{3} \mu, \\
U^{n}=\left(\begin{array}{l}
T^{n} \\
q_{x}^{n} \\
q_{y}^{n}
\end{array}\right), \quad A_{0}=\left(\begin{array}{ccc}
\rho c_{p} & 0 & 0 \\
0 & 0 & 0 \\
0 & 0 & 0
\end{array}\right) \\
A_{1}=\left(\begin{array}{ccc}
\rho c_{p} u & -k & 0 \\
-1 & 0 & 0 \\
0 & 0 & 0
\end{array}\right) \\
A_{2}=\left(\begin{array}{ccc}
\rho c_{p} v & 0 & -k \\
0 & 0 & 0 \\
-1 & 0 & 0
\end{array}\right) \\
A_{3}=\left(\begin{array}{ccc}
0 & 0 & 0 \\
0 & 1 & 0 \\
0 & 0 & 1
\end{array}\right)
\end{array}
$$

Assumed solution approach or the trial function of Eq. (3) is

$$
U^{n}=\sum_{i=1}^{m} \psi_{i}(x, y)\left(\begin{array}{c}
\left(T^{n}\right)_{i} \\
\left(q_{x}^{n}\right)_{i} \\
\left(q_{y}^{n}\right)_{i}
\end{array}\right)
$$

With $\psi(x, y)$ is a linear interpolation. Then the residue is obtained

$$
I(u)=\int_{\Omega}\{A \mu-F\}^{2} d \Omega
$$

In order that $\mu$ can minimize function I in Equation (4), for the test function $V=\psi_{i}\left(\begin{array}{l}1 \\ 1 \\ 1\end{array}\right)$ must meet

$$
\lim _{t \rightarrow 0} \frac{d}{d t} I(\mu+t V) \equiv 2 \int_{\Omega}(A V)^{T}(A \mu-F) d \Omega=0
$$
Or

$$
\int_{\Omega}(A V)^{T}(A \mu) d \Omega=\int_{\Omega}(A V)^{T} F d \Omega
$$

\section{CONJUGATE GRADIENT METHOD}

In general, finite element method will produce a system of equations

$$
K_{\alpha \beta} U_{\beta}=F_{\alpha}
$$

Iterative solutions can be obtained using MCG with the following steps:
a. Assume an approximate solution $U_{\alpha}^{r}$
b. Count residue $E_{\alpha}^{r}$

$$
E_{\alpha}^{r}=F_{\alpha}-K_{\alpha \beta} U_{\beta}^{r}
$$

c. Define auxiliary variables $P_{\alpha}^{r}$

$$
P_{\alpha}^{r}=E_{\alpha}^{r}
$$

d. Count residue $r$ th iteration

e. Count

$$
\bar{E}_{\alpha}^{r}=K_{\alpha \beta} P_{\beta}^{r}
$$

$$
a^{r}=\frac{E_{\alpha}^{r} P_{\alpha}^{r}}{\bar{E}_{\beta}^{r} P_{\beta}^{r}}
$$

f. Count solution at $(r+1)$ th iteration

$$
U_{\alpha}^{r+1}=U_{\alpha}^{r}+\alpha^{r} P_{\alpha}^{r}
$$

g. Count residue at $(r+1)$ th iteration

h. Count

i. Count auxiliary variables

$$
b^{r+1}=\frac{E_{\alpha}^{r+1} E_{\alpha}^{r+1}}{E_{\beta}^{r} E_{\beta}^{r}}
$$

$$
P_{\alpha}^{r+1}=E_{\alpha}^{r+1}+b^{r+1} P_{\alpha}^{r}
$$

j. Return to step d until the result obtained is convergent or when $E_{\alpha}^{r}$ already meet the tolerance value.

\section{SIMULATION OF DIFFUSION- CONVECTION IN TWO-DIMENSIONAL SPACE}

In the diffusion convection equation, if rate $u$ and $v$ are 0 which is mean there is no flow, it will be obtained conduction equation. For the example, a thin steel plate measuring $0.4 \mathrm{~m} \mathrm{x}$ $0.4 \mathrm{~m}$ which is perfectly insulated on its surface has an initial temperature of $100^{\circ} \mathrm{C}$. On the four sides are given a temperature of $0^{\circ} \mathrm{C}$ and $\mathrm{C}=1 \mathrm{~m} / \mathrm{s}$.

When resolved analytically using a separate variable method will be obtained solution [4]

$$
\begin{gathered}
u(x, y, t)=\frac{400}{\pi^{2}} \sum_{\substack{m=1,3,5, \ldots \\
n}}^{\infty} \sum_{n=1,3,5, \ldots}^{\infty} \frac{1}{m n} e^{-\left(\left(\frac{n \pi}{0.4}\right)^{2}+\left(\frac{m \pi}{0.4}\right)^{2}\right) t} \\
\sin \left(\frac{n \pi}{0.4} x\right) \sin \left(\frac{m \pi}{0.4} y\right)
\end{gathered}
$$

Furthermore, the problem is sought solution approach using method element to least square with MATLAB so obtained the following results

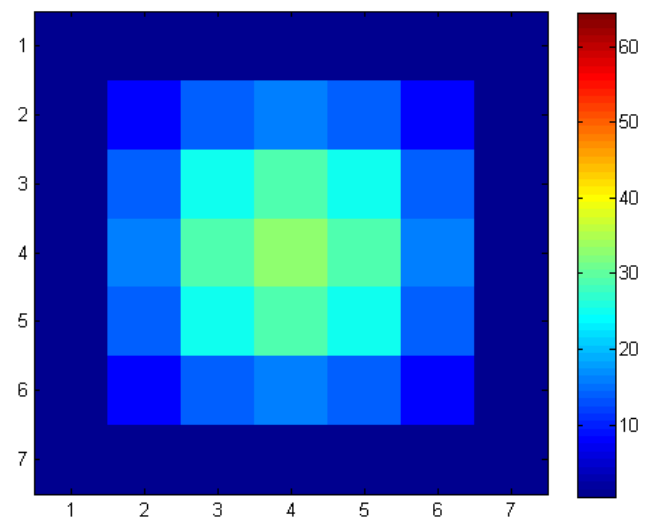

Figure 1. Approximation solution by taking $\Delta x=\Delta y=$ $\frac{0.4}{6}$ and $\Delta t=0.00025$ when $t=0.0025$ 


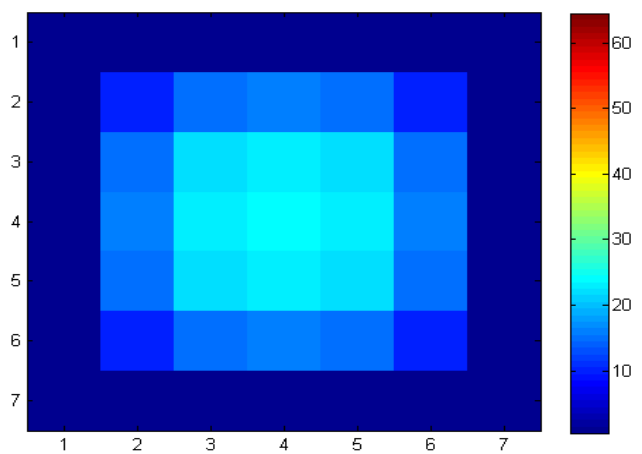

Figure 2. Analytical solution by taking $\Delta x=\Delta y=\frac{0.4}{6}$ and $t=0.0025$

In the process of calculating the solution approach, for each iteration requires the value $\frac{\partial^{2} T}{\partial x^{2}}$ and $\frac{\partial^{2} T}{\partial y^{2}}$ on each node but because there is no information in the problem then use finite different method (center) to find the 2nd partial derivative on each node. From both figures it appears that there is an error especially on the middle node which is the biggest error value compared to other nodes with average error 1.3171

Then given another example of diffusion convection equation in two-dimensional space. A system with a length of 1 unit and a width of 1 unit or $0 \leq x \leq 1$ and $0 \leq y \leq 1$ with an initial temperature of $10^{\circ} \mathrm{C}$ as well on the left and above the temperature is fixed $40^{\circ} \mathrm{C}$. Furthermore, the rate flow rate of $u=0,02$ and $v=-0,01$ and $\rho c_{p}=$ 1J $/ \mathrm{m}^{3} \mathrm{C}$.

$$
\frac{\partial T}{\partial t}+0,02 \frac{\partial T}{\partial x}+(-0.01) \frac{\partial T}{\partial y}=k \frac{\partial^{2} T}{\partial x^{2}}+k \frac{\partial^{2} T}{\partial y^{2}}
$$

The boundary condition of the system is

$$
\begin{aligned}
& T(0, y, t)=40 \\
& T(x, 1, t)=40
\end{aligned}
$$

$\frac{\partial T}{\partial x}=0 \quad$ on $x=1$

$\frac{\partial T}{\partial y}=0 \quad$ on $y=0$

Next by taking $\Delta t=0.1$ and taken $t=50$ results obtained as following

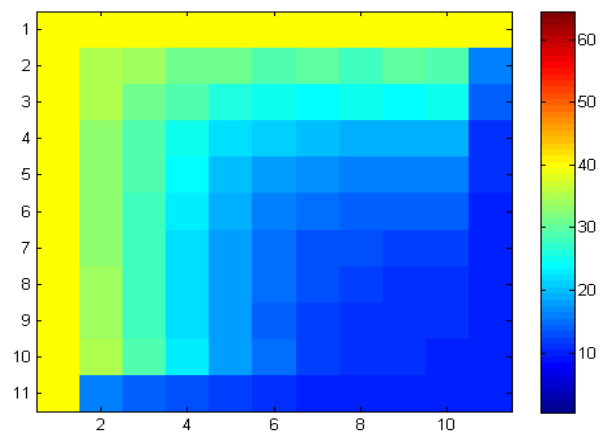

Figure 3. Approximation solution for $k=1$

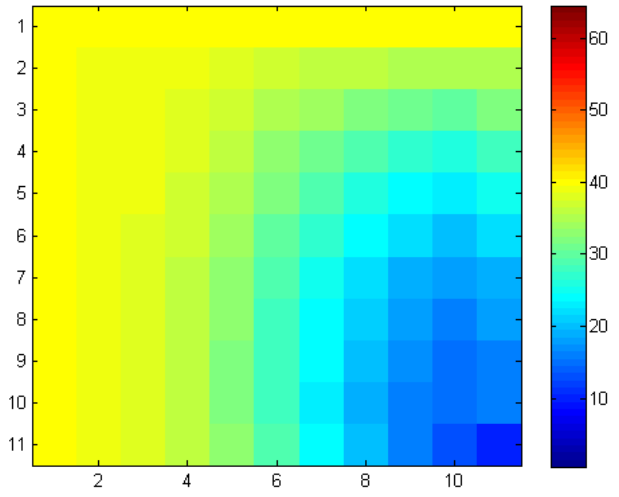

Figure 4. Approximation solution for $k=0.01$

In Figs (4) and (5) it is seen that by decreasing the value of $k$ the rate of temperature change in each node becomes larger.

\section{CONCLUSION}

The diffusion convection equation in two dimensional space

$$
\rho c_{p}\left(\frac{\partial T}{\partial t}+u \frac{\partial T}{\partial x}+v \frac{\partial T}{\partial y}\right)=k\left(\frac{\partial^{2} T}{\partial x^{2}}+\frac{\partial^{2} T}{\partial y^{2}}\right)
$$

which is a 2 nd order differential equation, can always be converted into 1 st order differential equations system. After being discredited by the time variable using $\theta$ method, then solved by using Least Square finite element method to obtained linear equation system in matrix form

$$
K U=F
$$

\section{REFERENCES}

[1] Bejan, A., 2013, Convec-tion Heat Transfer, Wiley, and Sons.

[2] Jiang, B.N., 1998, The Least Squares Finite Element Method, Springer-Verlag, Berlin.

[3] Jiji, L.M., 2006, Heat Convection, Springer-Verlag, Berlin.

[4] Kumar, A., Jaiswal, D.K., Kumar, N., 2009, Analytical Solutions of One-Dimensional Advection-Diffusion Equation with Variable Coefficients in Finite Do- main, Banaras Hindu University, India.

[5] Perko, L., 2000, Differential Equations and Dynamical Sys- tem, Springer-Verlag, New York.

[6] Pireira, V.D., Silva, J.B.C, Moura, L.F.M, dan Romao, E.C., 2014, Stabilized Least Squares Finite Element Method for 2D and 3D Convection-Diffusion, Wseas Transactions on Applied and Theoretical Mechanics, Vol. 9, hal. 45-59, 2014 